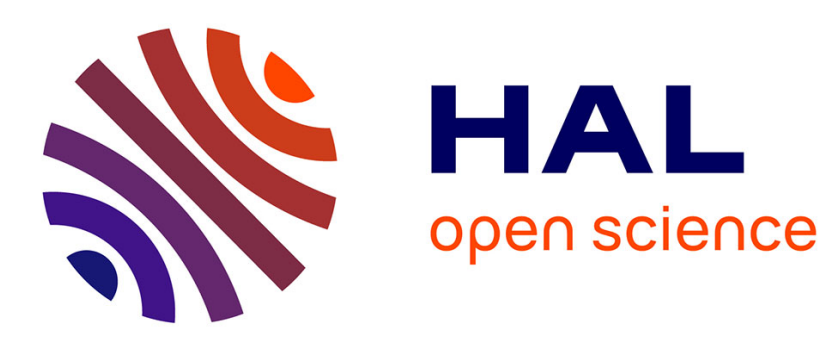

\title{
Les jeunes, leurs enseignants et Wikipédia : représentations en tension autour d'un objet documentaire singulier
}

Gilles Sahut

\section{- To cite this version:}

Gilles Sahut. Les jeunes, leurs enseignants et Wikipédia: représentations en tension autour d'un objet documentaire singulier . Documentaliste - Sciences de l'Information, 2014, 51 (2), pp.70-79. sic_01359610

\author{
HAL Id: sic_01359610 \\ https://archivesic.ccsd.cnrs.fr/sic_01359610
}

Submitted on 2 Sep 2016

HAL is a multi-disciplinary open access archive for the deposit and dissemination of scientific research documents, whether they are published or not. The documents may come from teaching and research institutions in France or abroad, or from public or private research centers.
L'archive ouverte pluridisciplinaire HAL, est destinée au dépôt et à la diffusion de documents scientifiques de niveau recherche, publiés ou non, émanant des établissements d'enseignement et de recherche français ou étrangers, des laboratoires publics ou privés. 


\title{
Les jeunes, leurs enseignants et Wikipédia : représentations en tension autour d'un objet documentaire singulier
}

\author{
Gilles SAHUT, Université de Toulouse, LERASS, gsahut@univ-tlse2.fr
}

Depuis sa création il y a douze ans, Wikipédia a provoqué - et provoque encore - maints débats et prises de position. L'étude des opinions et, plus largement, des représentations ${ }^{1}$ attachées à cette encyclopédie si particulière paraît relever de plusieurs enjeux. Tenter de les cerner revient à se pencher sur la circulation sociale d'un objet documentaire inédit qui s'inscrit dans la tradition encyclopédique mais résulte de modalités de production radicalement nouvelles. Au début des années 2000, alors que certaines encyclopédies proposaient une version sur cédérom, Juanals avait mis en évidence les mutations des représentations attachées à ce type de document. Leurs utilisateurs accordaient une faible valeur symbolique au support électronique qui tranchait avec l'affirmation de sa valeur d'usage [11]. Quelle peut être alors la légitimité d'une encyclopédie numérique qui n'est plus écrite par des experts clairement identifiés et à laquelle tout le monde peut participer ?

Régulièrement, les médias se font l'écho de problèmes liés à Wikipédia (erreurs, manipulations, plagiats, etc.). Les acteurs des institutions de savoir traditionnelles (l'école, la bibliothèque) ont souvent affiché de la suspicion, voire une franche hostilité à son égard [8]. Ceci n'a pas empêché l'encyclopédie collaborative de devenir le sixième site le plus consulté au monde. L'enquête récente menée aux États-Unis auprès de 2.462 enseignants du secondaire illustre bien le paradoxe qu'elle génère. Si 87 \% d'entre eux disent avoir recours à l'encyclopédie, ils sont également nombreux qui déclarent déconseiller, voire interdire son usage aux élèves [18]. L'étude des représentations autour de Wikipédia peut constituer une entrée pour tenter de comprendre pourquoi cette source est à la fois si controversée et autant utilisée. Elle sous-tend également un questionnement sur l'acceptabilité documentaire $^{2}$ de Wikipédia. Nous faisons référence ici au fait qu'il existe des valeurs

\footnotetext{
${ }^{1}$ Le terme de "représentation " peut renvoyer à des significations différentes selon les contextes et les disciplines. On considérera ici que les représentations sont des images mentales d'un objet pour un individu ou un groupe social. Elles comportent une dimension cognitive (ce sont des savoirs communs ayant une visée pratique et permettant d'interpréter le réel) et sociale (leur signification est construite et partagée au sein d'un contexte socio-culturel donné). Elles sont composées d'idées, de croyances, d'opinions. (Pierre Mannoni, Les représentations sociales, PUF, 2012).

${ }^{2}$ Le concept d'acceptabilité provient des études sur les interactions homme-machine et ergonomie des logiciels. Comme I'utilité et I'utilisabilité, il est une composante de l'évaluation des technologies numériques. II correspond aux
} 
partagées par une communauté qui reflètent une hiérarchie des sources et oriente la décision d'admettre ou non leur utilisation. L'acceptabilité documentaire correspondrait à l'idée que, selon les contextes, la citation d'une source sera jugée acceptable, discutable ou non pertinente. En lien avec cela, il faut noter que la majorité des études en sciences de l'information et/ou de la communication publiées sur les usages et représentations de Wikipédia portent sur des jeunes scolarisés dans l'enseignement secondaire ou supérieur. Cela ne tient pas seulement au fait que ce public est aisément accessible pour les chercheurs. Cette focalisation s'explique aussi par l'existence d'enjeux théoriques et d'implications pratiques concernant les enseignants et les professionnels de l'informationdocumentation exerçant en milieu scolaire ou universitaire. D'une part, ces recherches peuvent aider à comprendre pourquoi les jeunes font un usage massif de Wikipédia, parfois au détriment des ressources physiques ou virtuelles offertes par les espaces documentaires institutionnels. D'autre part, elles sont susceptibles d'être mobilisées afin d'alimenter la réflexion sur l'éducation à la culture informationnelle devant être dispensée par les établissements du secondaire et du supérieur. Après la synthèse de ces recherches, nous présentons la méthodologie et les résultats d'une enquête que nous avons menée auprès de jeunes âgés de 11 à 25 ans.

\section{1 État de la question}

Que ce soit en France ou dans d'autres pays, de nombreuses études attestent de l'usage fréquent de Wikipédia en rapport avec la scolarité ou avec la vie quotidienne $[1,3,6]$. Formuler une requête sur Google et activer le lien menant à un article de Wikipédia est devenu une routine caractérisant nombre de comportements informationnels. En effet, les internautes ont tendance à juger les pages de Wikipédia plus pertinentes que celles des autres sites figurant dans la liste des résultats [15]. C'est donc que l'encyclopédie bénéficie de représentations positives qui conduisent à sa consultation.

Que ce soit au lycée [22] ou à l'université [10, 19], Wikipédia est perçue comme particulièrement utile lors de la phase initiale de recherche d'information car elle autorise une vue d'ensemble du sujet afin de le cerner et de mieux le comprendre. En ce sens, elle peut être qualifiée d'outil de «pré-recherche»[10] et assure là les fonctions traditionnelles

représentations qu'a un individu de la valeur d'un système ou d'une technologie et à sa volonté d'utiliser ou non le système. L'acceptabilité est influencée par des valeurs, des affects et par le contexte social dans lequel l'outil technologique s'insère. (F. Février. Vers un modèle "expérience-acceptation ». Thèse en psychologie cognitive. Université de Rennes 2 , 2011). 
dévolues à une encyclopédie dans le processus de recherche d'information. Les jeunes attribuent d'autres caractéristiques positives à cette source : sa complétude car elle est susceptible d'offrir des informations portant sur une gamme extrêmement large de sujets [10, $19,17,1]$; son actualité car elle est plus rapidement modifiable qu'une ressource imprimée [10] ; le fait qu'elle soit compréhensible puisque les élèves ou étudiants estiment qu'elle est adaptée à leur niveau [22; 10]. Ils considèrent également que l'encyclopédie est rapidement accessible et facilement exploitable, la navigation en son sein étant fréquemment qualifiée d'aisée et la présentation des articles, particulièrement claire [10, 19, 17, 1]. En ce sens, le terme anglais de convenience - que l'on peut traduire par commodité d'usage - paraît particulièrement approprié pour qualifier Wikipédia [3].

Celle-ci fait toutefois l'objet de jugements moins favorables. Une enquête menée auprès d'adolescents américains âgés de 11 à 18 ans indique ainsi que $43 \%$ d'entre eux n'accordent qu'une confiance limitée aux articles de Wikipédia alors qu'ils sont près d'un tiers à leur accorder un grand crédit [6]. D'autres études soulignent que l'encyclopédie est dotée d'une confiance moyenne [10;16]. Ainsi, chez les lycéens canadiens étudiés par Julien et Barker, son usage génère une “tension inquiète »: le recours à Wikipédia est fréquent mais, paradoxalement, certains d'entre eux ne la reconnaissent pas comme une source fiable [13]. Une recherche conduite auprès d'étudiants de Hong Kong apporte sur ce point un éclairage différent : le fait que l'encyclopédie donne accès à des informations jugées utiles pour des usages académiques a une incidence positive très nette sur la confiance qui lui est accordée [19].

Cet état de la question nous amène aux constats et interrogations qui ont guidé notre travail. On remarque tout d'abord que toutes les études précédemment citées portent sur un seul niveau de scolarisation (par exemple, les lycéens ou les étudiants de licence), voire deux (par exemple, les collégiens et lycéens). De manière plus générale, c'est également le cas de la grande majorité des recherches sur les pratiques informationnelles des jeunes. Ce type de focalisation permet certes une précision de l'analyse mais ne favorise pas une approche différenciatrice selon le critère de l'âge et le niveau de scolarité. L'un des objectifs de notre recherche est donc de cerner la diversité des représentations et attitudes vis-à-vis de Wikipédia chez les collégiens, lycéens et étudiants et d'opérer des comparaisons selon cette variable. Plus précisément, nous cherchons à identifier les évolutions de la confiance octroyée à l'encyclopédie en fonction de sa fréquence d'utilisation, du contexte d'usage envisagé et du niveau de scolarité.

La comparaison entre les différents âges de la jeunesse est rendue possible par le fait que l'encyclopédie est largement connue et reconnue. À l'instar de Google, elle peut être considérée comme une « marque » qui, en tant que telle, est l'objet d'une réputation [1,9]. 
La prise en compte de ce facteur paraît primordiale quand on s'intéresse à la confiance accordée à une source d'information. Celle-ci relève de phénomènes sociaux au sens où elle dépend - au moins partiellement - des interactions entre individus et de l'appartenance à des communautés « qui partagent un ensemble de règles quant à la qualité des processus de vérification et de validation de l'information » [20]. Ainsi, une recommandation émise par une personne de confiance est susceptible d'avoir une influence sur la confiance attribuée à une source d'information et donc sur les choix effectués par un individu en quête d'information. Le phénomène inverse peut également se produire. Dans la littérature empirique, on repère ainsi des élèves ou étudiants qui font état de jugements négatifs sur Wikipédia émanant de leurs enseignants (dont, en France, les professeurs-documentalistes) [10, 17; 4]. Ceci nous amène à interroger les jeunes sur le rôle qu'ils assignent à l'encyclopédie dans le cadre de recherches prescrites devant aboutir à une production documentaire (dossier, mémoire, etc.). Dans ce cas de figure, la recherche d'information est intégrée à une situation de communication où les enseignants sont à la fois destinataires et évaluateurs du travail réalisé. Les élèves ou étudiants doivent donc non seulement construire une représentation des attentes des enseignants sur la production à réaliser mais aussi sur la nature des sources pouvant être utilisées pour y parvenir, et donc sur l'acceptabilité documentaire de Wikipédia.

\section{Cadre méthodologique}

Notre volonté de cerner les représentations sur Wikipédia nous a amené à choisir le questionnaire comme outil de recueil de données. Certes, ce procédé ne permet pas de saisir les pratiques informationnelles de manière fine et contextualisée parce qu'il peut exister un décalage entre les données déclaratives des répondants et les pratiques réellement mises en œuvre. II demeure cependant l'une des méthodes mobilisables afin d'appréhender les opinions, discours et représentations qui forment une part importante de la réalité sociale [21]. Nous avons formulé une majorité de questions fermées qui facilitent l'expression de réponses précises et se prêtent plus aisément au traitement statistique. Néanmoins, des questions ouvertes ont été également été retenues afin de recueillir des représentations plus riches et diversifiées. Les données qualitatives ainsi obtenues ont été recodées manuellement afin de les quantifier et de sélectionner les verbatims que nous rapportons dans la partie consacrée aux résultats. 
Le questionnaire a été administré en ligne de décembre 2012 à septembre 2013 dans six collèges, quatre lycées (deux lycées d'enseignement général, deux lycées professionnels) ainsi qu'auprès d'étudiants suivant un cursus bac +2 ou bac +3 (BTS comptabilité, DUT documentation et informatique, licence arts appliqués, art et communication) et en masters conduisant aux métiers de l'enseignement dans les différentes disciplines scolaires. Pour les établissements du second degré, ce sont des professeurs-documentalistes qui ont encadré la passation du questionnaire par des classes ou demi-classes, en salle informatique ou au CDI. Afin de minimiser les biais pouvant être générés par la présence d'enseignants, les élèves ont été informés oralement et par écrit que l'enquête se situait dans le cadre d'une recherche scientifique, qu'elle était donc anonyme et que leurs enseignants n'auraient pas connaissance des résultats. Dans l'enseignement supérieur, ces informations ont été reprises dans un courrier électronique signalant le questionnaire aux étudiants. Pour ce niveau de scolarité, le taux de répondants est estimé à $15 \%$. Au final, nous avons analysé les réponses de 841 jeunes (dont 54,1\% de filles), soit 256 collégiens, 265 lycéens, 148 étudiants suivant un cursus bac +2 ou bac +3 et 172 étudiants de master.

\section{Présentation et interprétation des résultats}

Nous pouvons tout d'abord confirmer la très forte notoriété de Wikipédia. En effet, seuls $1,8 \%$ des jeunes interrogés déclarent ne pas connaître l'encyclopédie ${ }^{3}$, ce pourcentage étant comparable à ceux relevés par d'autres enquêtes aux États-Unis ${ }^{4}$. Les usages de Wikipédia sont largement répandus puisque 94,3\% de ceux qui connaissent l'encyclopédie en sont également des utilisateurs. À titre de comparaison, en 2008, 76 \% des Français âgés de 18-24 ans déclaraient avoir visité ce site [7]. La proportion de jeunes utilisateurs de Wikipédia a donc augmenté au cours de ces dernières années en France, tendance qui se retrouve également dans la population estudiantine d'autres pays [12]. Toutefois, notre enquête met en évidence des écarts notables pour ce qui est de sa fréquence d'utilisation. En effet, celle-ci peut être mensuelle, hebdomadaire ou quasi-quotidienne (fig.1). On relève

\footnotetext{
${ }^{3}$ Ces répondants ont été exclus de la suite du questionnaire qui ne s'adresse donc qu'aux jeunes connaissant Wikipédia.

${ }^{4} 3 \%$ dans [10], $1 \%$ dans [6]
} 
aussi qu'elle s'intensifie en fonction des niveaux de scolarisation et de l'âge. Elle est en moyenne plus élevée à l'université qu'en collège ou lycée ${ }^{5}$.

Figure 1: Fréquence d'utilisation de Wikipédia

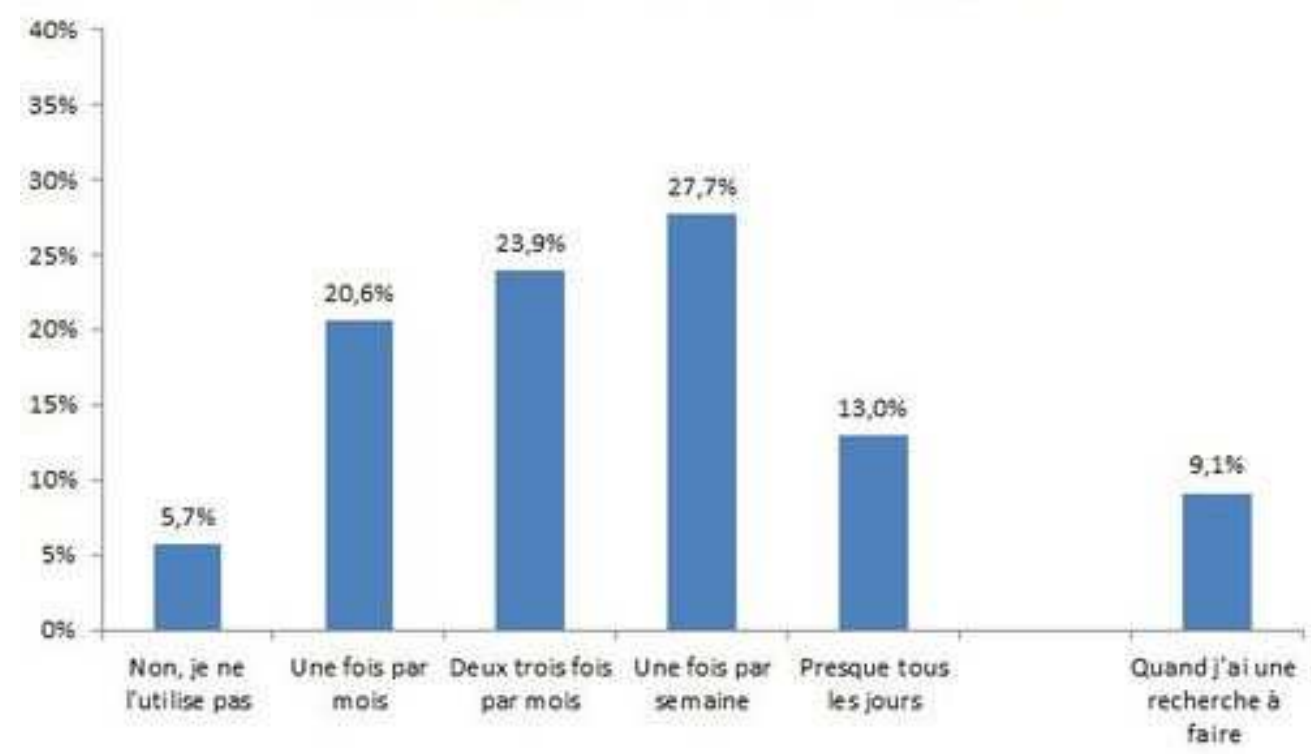

\section{Des représentations contrastées}

Si on excepte une petite minorité - autour de $6 \%$ - qui paraissent réfractaires à son utilisation, Wikipédia est considérée comme une ressource utile $(48,1 \%)$, voire très utile $(46,3 \%)$. Quand ils se fondent sur leur expérience passée, les jeunes formulent des jugements globalement positifs à propos de la qualité de l'information disponible sur Wikipédia et ce, quel que soit leur niveau de scolarité. Le point de vue majoritaire est que l'encyclopédie collaborative leur a permis d'accéder le plus souvent à des informations utiles (93\%), compréhensibles (92\%) et exactes (92,7\%). À l'instar des étudiants américains interrogés par Lim [16], la plupart des répondants évaluent positivement leur expérience de consultation de Wikipédia.

Si la valeur d'utilité de Wikipédia est majoritairement avérée, qu'en est-il de la confiance qui lui est accordée ? Pour des recherches d'information liées à l'enseignement, la confiance affichée vis-à-vis de l'encyclopédie collaborative est inférieure à celle des sources

\footnotetext{
${ }^{5} \mathrm{Si}$ on effectue une moyenne en se fondant sur une graduation de la fréquence d'utilisation (de 1 pour une non utilisation à 5 pour une utilisation quasi-quotidienne), on obtient une moyenne de 2,83 pour les collégiens, 3,13 pour les lycéens, 3,69 pour les étudiants de bac $+2 /+3$ et 3,58 pour ceux de master.
} 
agréées par les enseignants et, plus globalement, par l'institution (manuels scolaires, revues de $\mathrm{CDI}$ et de bibliothèque notamment) (fig.2) ; ce qui, d'une certaine manière, confirme la prédominance toujours actuelle de la culture de l'imprimé dans l'univers scolaire [2]. Pour les recherches en rapport avec les loisirs, les jeunes se montrent plus confiants envers des sources non institutionnelles telles que l'entourage familial ou amical et les sites repérés grâce à Google (fig.3). La prise en compte du niveau de scolarité amène à nuancer ces affirmations. Au collège, la confiance envers Wikipédia est plus élevée pour les recherches académiques que pour celles concernant les loisirs. Ce n'est plus le cas pour les autres niveaux de scolarité et, en particulier, pour les étudiants de master qui font preuve d'une plus grande défiance vis-à-vis de l'encyclopédie collaborative pour la recherche d'information en rapport avec l'enseignement (fig.4).

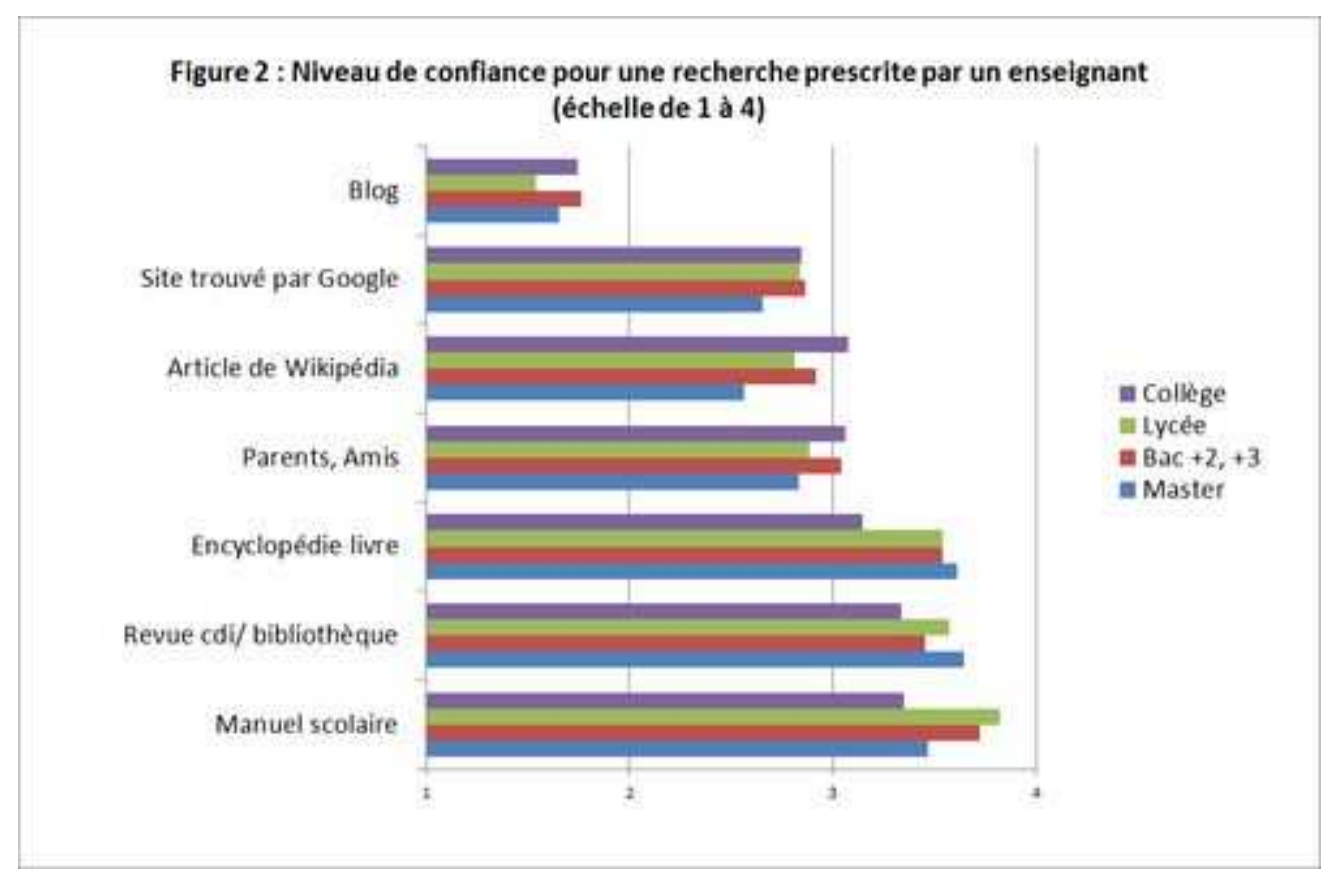




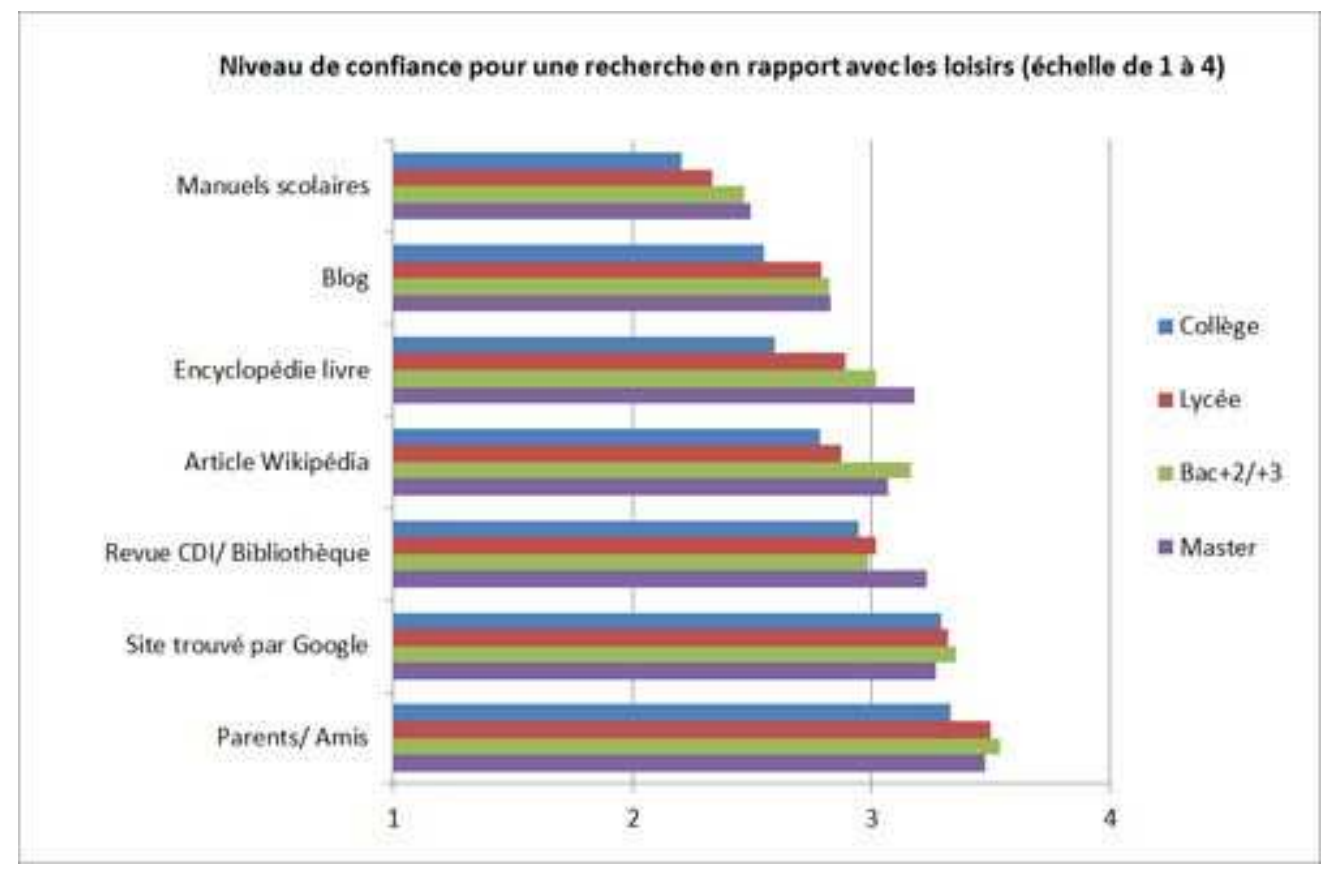

Figure 4 : Niveau de confiance accordé à Wikipédia selon la nature de la recherche (échelle de 1à 4)

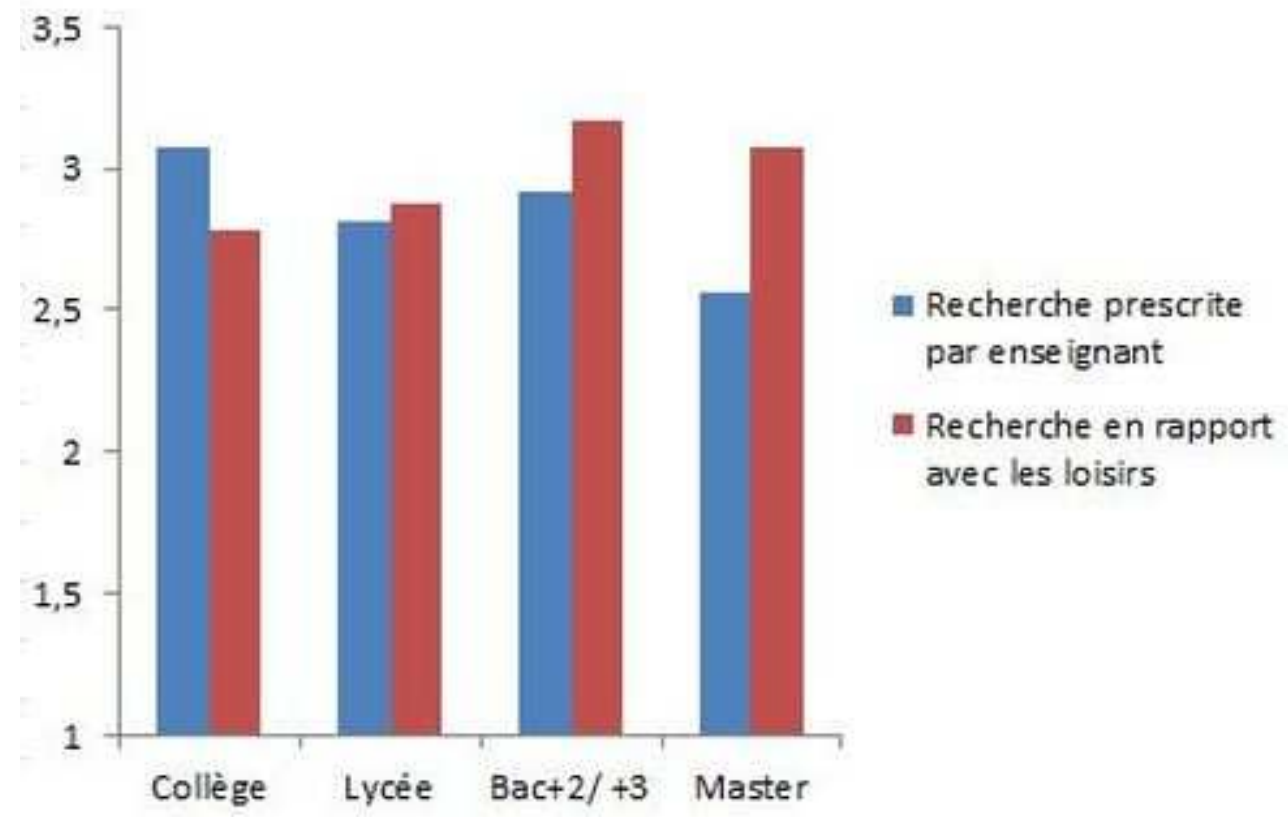

Les commentaires aux questions ouvertes à propos de l'utilité de Wikipédia ont été recodés et regroupés selon une démarche inductive, ce qui nous a permis d'identifier trois types de jugements (positifs, négatifs, conditionnels) portés sur l'encyclopédie. 


\section{- Les jugements positifs}

Les jugements positifs peuvent être à leur tour subdivisés en deux catégories : ceux qui correspondent à des qualifications positives de l'encyclopédie et ceux qui soulignent l'utilité des fonctions de Wikipédia. Pour les premiers, l'orientation sémantique de l'opinion s'exprime généralement au travers d'adjectifs qui s'apparentent pour la plupart à des critères d'évaluation utilisés lors de la recherche d'information ${ }^{6}$ (fig.5). Les deux qualifications positives les plus fréquemment citées sont la complétude de Wikipédia et sa commodité d'usage et ce, quel que soit le niveau d'étude. Wikipédia est en effet perçue comme une source exhaustive, susceptible d'apporter des informations sur une large gamme de sujets. “On y trouve tout » ou « presque tout », affirment nombre de répondants. Sa commodité d'usage est mise en exergue : l'encyclopédie est appréhendée comme un moyen simple et rapide d'accéder à une information facilement repérable et utilisable. Ces perceptions sont analogues à celles évoquées dans d'autres recherches menées sur la base de questionnaires et/ou d'entretiens [10, 1, 3]. En revanche, l'actualisation de l'information, considérée comme l'un des atouts majeurs de l'encyclopédie collaborative dans l'enquête de Head et Eisenberg [10], n'est mentionnée que par une infime partie des jeunes interrogés $(1,6 \%)$ et ne semble donc pas être un critère prédominant pour sa consultation. Peu d'entre eux mettent en exergue sa fiabilité $(8,3 \%)$ et un nombre encore plus réduit $(3,2 \%)$ témoigne d'une adhésion aux fondements mêmes du projet wikipédien : libre accès aux connaissances, échange et collaboration entre internautes, multiplicité des points de vue.

\footnotetext{
${ }^{6}$ Pour une vision d'ensemble de ces critères, on peut se référer au tableau situé p. 103 de l'ouvrage Qu'est-ce que rechercher de l'information ? N. Boubée et A Tricot, Presses de l'Enssib, 2010.
} 


\section{Figure 5 Qualifications positives de Wikipédia}

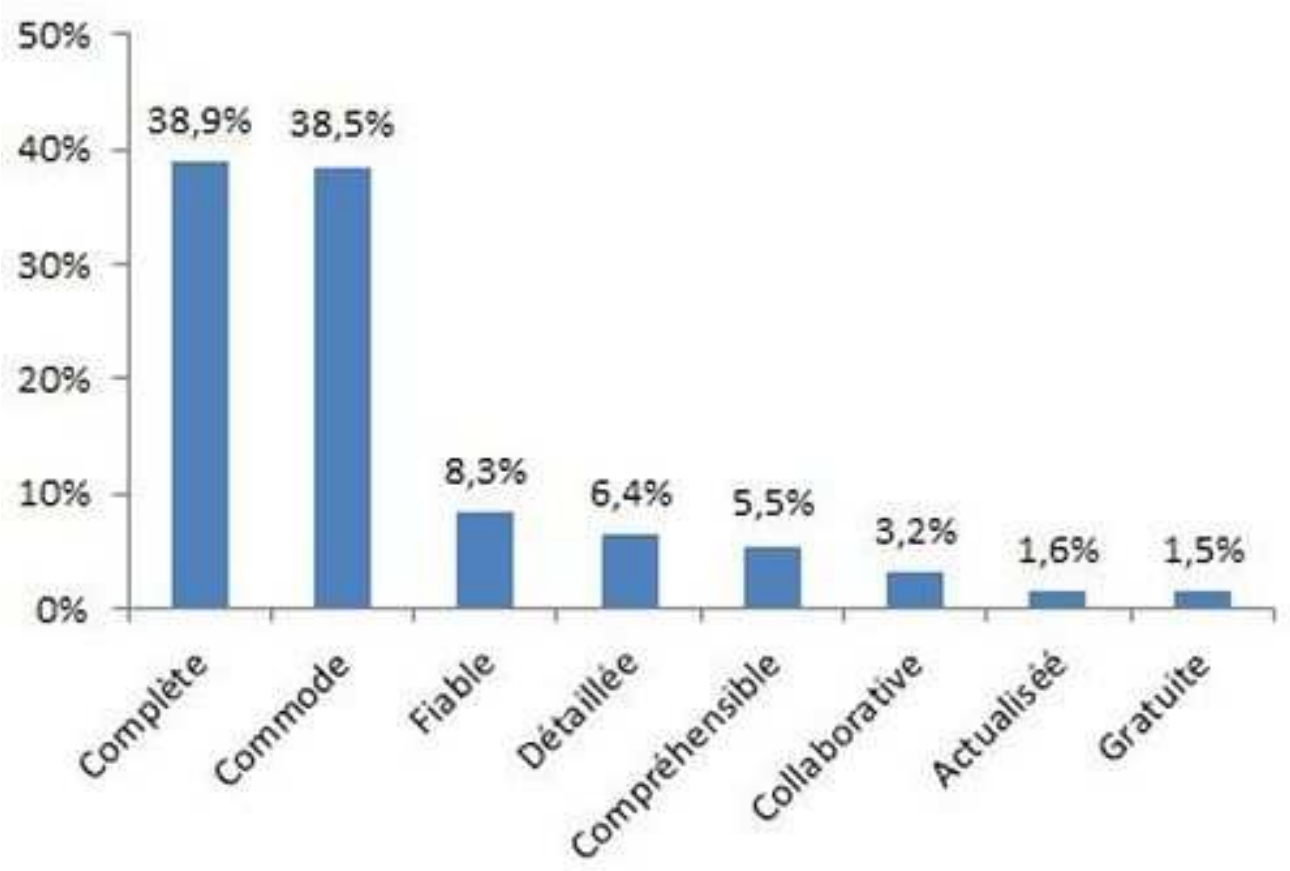

Le second type de jugements positifs exprime l'intérêt attribué à des fonctions de Wikipédia (fig.6). L'encyclopédie est particulièrement appréciée pour la réalisation de travaux scolaires ou universitaires et pour la recherche de définitions ou de biographies qui peuvent faire également partie des tâches prescrites par les enseignants. Pour 5,8 \% des répondants seulement, l'encyclopédie est dotée d'une valeur positive au sens où elle fait figure de source « instructive », " susceptible d'étancher la soif intellectuelle » ; " On peut y apprendre plein de choses » et ce, même en dehors du cadre scolaire. Cette minorité de jeunes attribue à l'encyclopédie collaborative une valeur cognitive et culturelle traditionnellement dévolue au support livre. Ceci fait écho aux travaux de Le Douarin et Delaunay-Téterel qui ont repéré chez les lycéens une diversité de stratégies d'utilisation des ressources numériques (dont Wikipédia). Celles-ci ne sont pas seulement instrumentalisées afin de faire face aux exigences scolaires mais elles peuvent également être mobilisées pour renforcer les connaissances et même alimenter un intérêt pour les domaines enseignés [14].

Certaines des fonctions de Wikipédia sont significativement corrélées au niveau de scolarité. Son utilité pour les tâches prescrites par les enseignants est mentionnée par près d'un tiers des collégiens mais seulement par 3,1\% des étudiants de master. Ainsi, dans les commentaires, de nombreux collégiens perçoivent Wikipédia comme une « aide » - terme souvent employé - à la réalisation de recherches ou devoirs scolaires. Certains n'hésitant pas, à l'instar d'une élève de $6^{\mathrm{e}}$, à lui accorder un rôle exclusif : " C'est le seul site qui nous 
sert à faire des exposés ». À l'inverse, Wikipédia n'est signalé comme un outil de prérecherche qu'à partir du lycée $(6,3 \%)$ et plus encore en licence $(21 \%)$ et en master $(41,1$ $\%)$. S'y référer permet de "se faire une idée du sujet » et d'exploiter les références des documents placés en fin d'article. On discerne bien ici les différences de représentations attachées à Wikipédia. Pour nombre de collégiens, l'encyclopédie fait figure d'adjuvant indispensable à la recherche d'information. L'article de Wikipédia - ou, parfois, le court résumé par lequel il débute - leur semble en adéquation avec les attentes de l'enseignant prescripteur de recherche documentaire. II peut s'apparenter à un « document parfait 》 particulièrement prisé par les élèves car paraissant répondre à l'ensemble des questions qu'ils se posent [23]. À l'opposé, les étudiants de master soulignent que l'encyclopédie ne peut faire office de source unique, voire pour certains, de « vraie source », ce qui peut se comprendre si on examine les qualifications négatives dont Wikipédia fait l'objet.

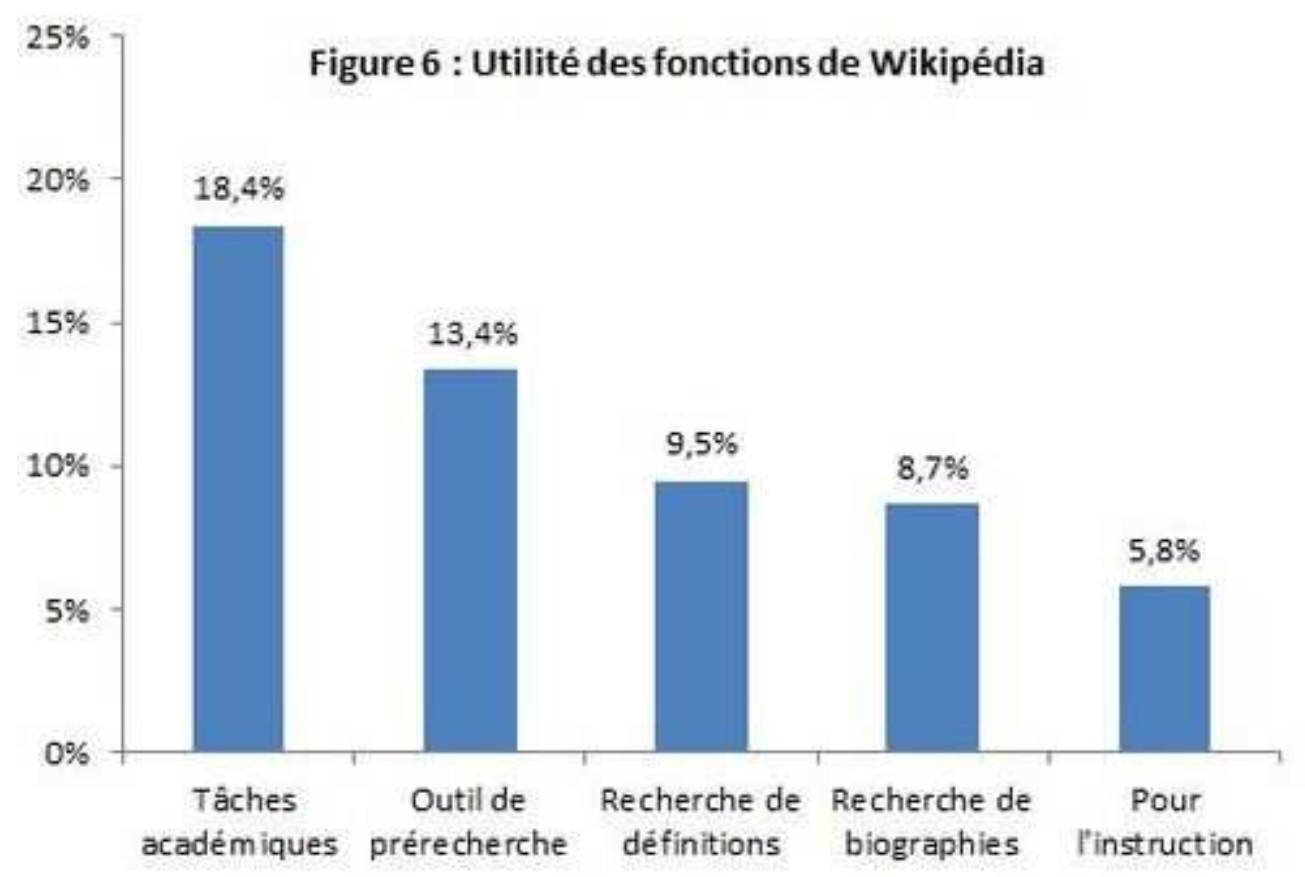

\section{- Les jugements négatifs}

Les opinions négatives dominantes sur Wikipédia relevées dans notre étude sont, d'une part, son manque de fiabilité entendu ici comme la présence avérée d'erreurs ou, plus globalement, comme une incertitude planant sur l'exactitude des articles et, d'autre part, une vision dépréciative de la possibilité offerte à tout un chacun d'y contribuer (fig.7). Ces deux appréciations sont associées de manière récurrente par une relation de cause à effet. "Les 
informations ne sont pas fiables puisque modifiables ou rajoutées par les internautes...méfiance » indique à ce sujet une étudiante de master. Ces types de jugement négatif peuvent expliquer la confiance moyenne accordée à Wikipédia évoquée plus haut. Ils confirment l'existence chez les jeunes d'un certain scepticisme à l'égard du mode d'édition collaboratif déjà identifié par ailleurs [6]. Toutefois, nous relevons que la fréquence de ces appréciations négatives est liée au niveau de scolarité. Celles-ci s'avèrent rares dans les premières années du collège pour devenir plus fréquentes à partir de la classe de troisième, et plus encore au lycée et à l'université. Beaucoup d'étudiants de master se distinguent ici en établissant une opposition entre Wikipédia et les sources scientifiques. On décèle dans leurs commentaires la capacité à différencier les sources selon leur degré de scientificité ainsi que l'intériorisation des exigences académiques liées à la réalisation d'un mémoire.

\section{Figure 7 : Qualifications négatives de Wikipédia}

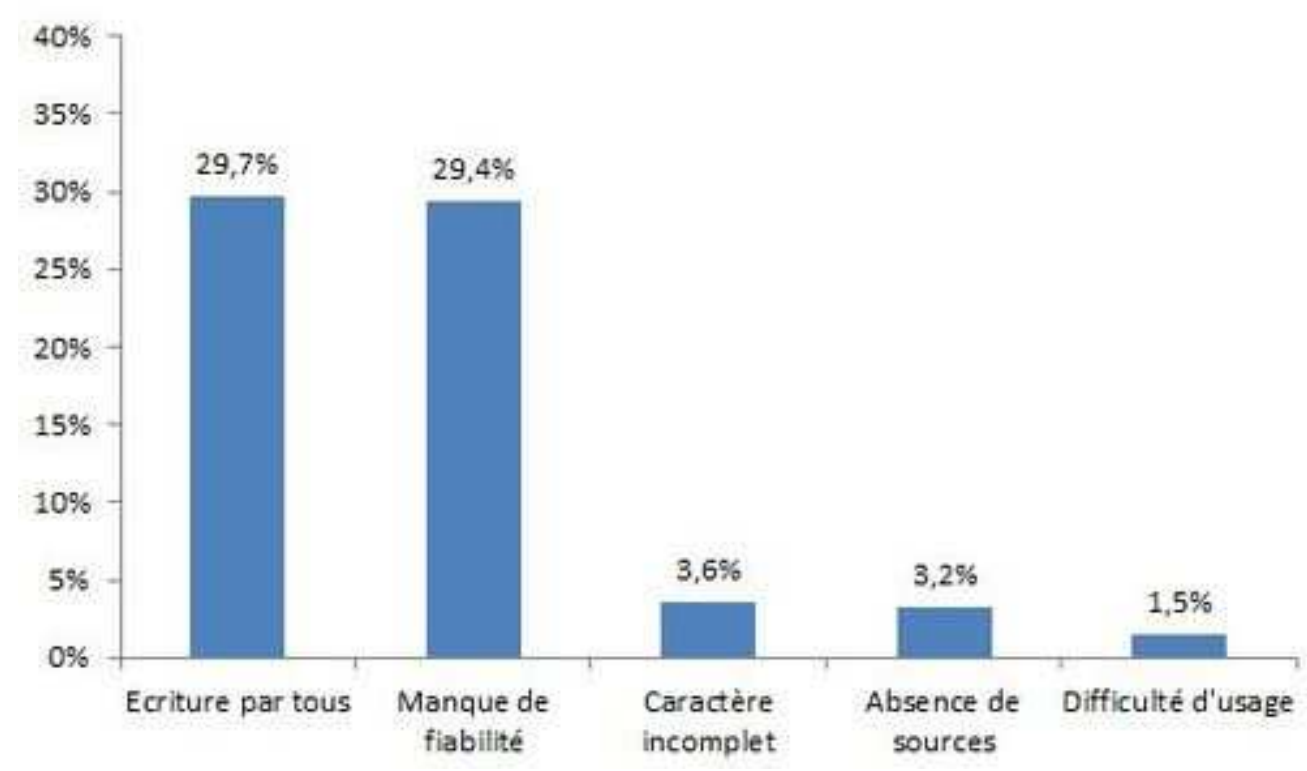

\section{- Les jugements conditionnels}

Ces types de jugement se distinguent des précédents car ils ne sont pas orientés positivement ou négativement. Ils indiquent que l'utilité de Wikipédia est subordonnée à une condition et correspondent à une formulation de type «Wikipédia n'est utile que si... ». Les énoncer revient à proposer une solution pouvant être adoptée afin de faire face à la perception d'un déficit de fiabilité de l'encyclopédie. Ainsi, vérifier les informations trouvées sur Wikipédia à partir d'autres sources d'information semble une condition nécessaire à son utilisation pour plus d'un jeune sur cinq, sans qu'il soit possible de savoir, dans le cadre de 
notre étude, si cette préconisation est véritablement appliquée lors de situations de recherche d'information. D'autres attitudes sont plus rarement évoquées. Certains répondants déclarent limiter l'utilisation de l'encyclopédie à des recherches " rapides ", « superficielles », « non scolaires » «pas scientifiques » (8\%) ou encore appeler à une certaine prudence vis-à-vis des informations qu'ils y trouvent $(5,8 \%)$. Là encore, la fréquence d'apparition de ces différents jugements conditionnels augmente progressivement du collège au master.

\section{Une réputation équivoque}

Notre enquête confirme que Wikipédia est l'objet de discours sociaux souvent dépréciatifs dans les sphères médiatiques, familiales, amicales ou éducatives. Une majorité des jeunes $(67,9 \%)$ ont déclaré avoir entendu ou lu des critiques négatives à propos de l'encyclopédie. De manière peu surprenante, elles portent essentiellement sur son manque de fiabilité ${ }^{7}$. À l'inverse, quatre jeunes sur dix disent avoir entendu des avis positifs sur Wikipédia, notamment dans l'entourage amical ou familial. Là encore, commodité d'usage et complétude sont les qualifications positives les plus souvent évoquées. Plus d'un jeune sur deux affirme avoir déjà entendu un de ses enseignants formuler une critique négative à l'égard de Wikipédia et seulement un cinquième d'entre eux des appréciations positives. Aussi, quand on les interroge sur la prise de position de leurs enseignants à l'égard de Wikipédia, il n'est pas étonnant de constater que les jeunes sont partagés. Selon une majorité d'entre eux, leurs enseignants ont une mauvaise opinion de Wikipédia mais la proportion d'avis contraires n'est pas pour autant négligeable ${ }^{8}$. II est à noter que, plus on avance dans la scolarité, plus les jeunes attribuent à leurs enseignants une image négative de l'encyclopédie collaborative. Alors que seuls $31,7 \%$ des collégiens pensent que leurs enseignants ont une mauvaise opinion de Wikipédia, 67,2 \% des lycéens, 68,2 \% des étudiants de licence et $76,7 \%$ des étudiants de master répondent en ce sens. Les doutes des jeunes sur l'acceptabilité documentaire de Wikipédia apparaissent clairement si on

\footnotetext{
${ }^{7} 41,2 \%$ des répondants disent avoir entendu une critique de cette nature.

${ }^{8}$ Selon $42,5 \%$ des jeunes interrogés, les enseignants ont une opinion plutôt négative de Wikipédia et pour 15,8 $\%$ très négative. Mais ils sont $36,2 \%$ à indiquer que leurs professeurs en ont sans doute une bonne et 5,2 \% une très bonne.
} 
examine leurs réponses quant à sa citation dans leurs travaux scolaires ou universitaires ${ }^{9}$. Seule une minorité (18\%) déclare la citer systématiquement, 50,3\% s'y refusent et 31,7\% indiquent que cela dépend de l'enseignant prescripteur. Autrement dit, pour la plupart d'entre eux, l'utilisation et/ou la citation de Wikipédia apparaît risquée car pouvant entraîner une dépréciation de leur production. Les étudiants de master se distinguent ici nettement de ceux de licence et des lycéens car ils se montrent généralement plus réticents à citer l'encyclopédie collaborative; ce qui peut indiquer à la fois une exigence accrue des enseignants en matière de recherche documentaire et/ou une conscience plus claire des normes académiques en vigueur (fig.8).

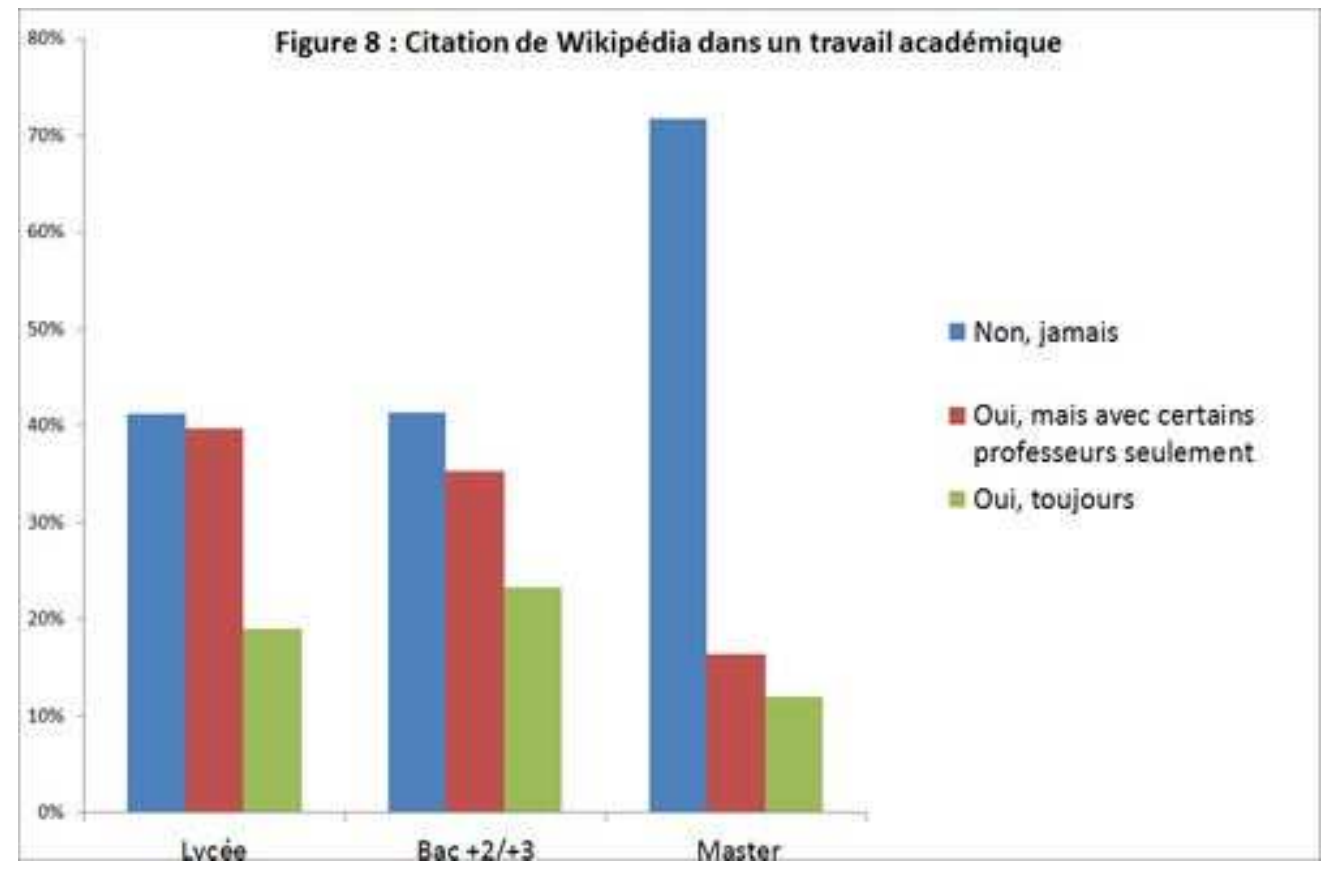

Les commentaires des répondants laissent entrevoir la diversité des stratégies mises en œuvre à l'occasion de recherches prescrites. Une stratégie d'évitement de Wikipédia tout d'abord. Elle est évoquée dans des déclarations de lycéens ou d'étudiants - jamais de collégiens - qui craignent que leurs enseignants aillent vérifier dans l'encyclopédie s'ils n'ont pas plagié un article. Cependant, au regard des recherches montrant unanimement la prégnance du couple Google-Wikipédia dans les pratiques informationnelles des jeunes, y compris pour les travaux académiques, il n'est guère probable que cet évitement soit très répandu. On peut en revanche supposer la mise en œuvre de stratégies de dissimulation de

\footnotetext{
${ }^{9}$ Nous avons ici exclu les réponses émanant des collégiens. La réalisation d'une bibliographie - ou plus largement d'une « documentographie » - ne semble pas être une pratique généralisée à ce niveau de scolarité. Les commentaires associés à cette question ont confirmé que certains collégiens ne la comprenaient pas.
} 
l'usage de Wikipédia, celui-ci étant perçu comme non souhaité, voire illégitime. Bien que difficilement avouable, ce type de pratiques affleure dans les commentaires. Quelques lycéens ou étudiants admettent le plagiat d'articles de l'encyclopédie lors de tâches prescrites ou l'attribuent à leurs pairs afin, peut-être, de s'en dédouaner. Certains disent ne pas citer Wikipédia afin d'éviter d'avoir une mauvaise note ou encore de « se faire engueuler » par des enseignants “d'une autre génération » qui, de toutes manières, "préféreront toujours les livres ». D'autres encore, davantage conscients des possibilités de contournement des exigences professorales, confessent citer les références bibliographiques présentes dans l'article de Wikipédia en lieu et place de celui-ci. Enfin, le fait qu'environ un tiers des répondants disent faire preuve d'un comportement citationnel différencié selon le professeur ne manque pas de surprendre. On discerne là une stratégie de repérage et de catégorisation de l'attitude des enseignants à l'égard de Wikipédia, éventuellement à partir des avis ou des consignes que celui-ci a pu donner ou encore à partir d'autres variables (son âge ? la discipline qu'il enseigne ?) qui restent dans notre étude à l'état de conjectures. De manière plus générale, ces réponses montrent que l'anticipation du jugement de l'enseignant est susceptible de peser sur les choix opérés par les jeunes en matière de sélection et citation des sources, essentiellement à partir du lycée.

Les commentaires permettent également de repérer la diversité de leurs réactions face aux opinions, réelles ou supposées, de leurs enseignants. Si la majorité des jeunes paraissent adhérer aux représentations dominantes sur le manque de fiabilité de Wikipédia, d'autres n'hésitent pas à les mettre en doute. II arrive ainsi que des lycéens ou étudiants dénoncent les lacunes d'une partie des professeurs dans le domaine de la culture numérique. " Il y a des statistiques qui montrent que le pourcentage d'erreur est faible, mais certains professeurs l'ignorent et ont Wikipédia en horreur " proclame une élève de terminale. Perce également chez d'autres la dénonciation d'une forme d'hypocrisie de la part d'enseignants qui bâtiraient leurs cours à partir de l'encyclopédie tout en refusant son utilisation à leurs élèves ou étudiants. Au travers de ces différents propos, on perçoit une remise en question de la relation pédagogique traditionnelle fondée sur une asymétrie entre des enseignants « dépositaires du savoir » et des élèves censés l'apprendre. Wikipédia est susceptible de faire vaciller l'autorité professorale au sens où sa facilité d'accès et d'usage peut faire croire à l'avènement d'un rapport au savoir plus égalitaire. Au lycée et plus encore à l'université, les quelques jeunes qui se présentent comme des contributeurs réguliers à l'encyclopédie ou disent adhérer aux valeurs de ce projet éditorial s'avèrent particulièrement soucieux de remettre en question les représentations négatives à son sujet et se montrent capables de développer une argumentation précise en faveur de sa fiabilité. "Mal vue par 
les vieux », Wikipédia paraît alors emblématique d'une culture numérique en décalage, voire en rupture, avec les normes de la culture scolaire traditionnelle. Son usage, source de tensions et d'incompréhensions, concourt à ce que Dioni a appelé une « fracture numérique scolaire » entre enseignants et apprenants [5].

\section{Conclusion}

Pour conclure, nous souhaitons préciser la notion d'acceptabilité documentaire en nous appuyant sur cette étude empirique. Elle résulte, en premier lieu, d'un rapport entre deux types de représentations : celles qui concernent la valeur informationnelle attribuée à un objet documentaire et celles portant sur les efforts générés par son utilisation. Selon cette perspective, Wikipédia est considérée comme une source apportant une information utile et complète sur une très large gamme de sujets. Facilement accessible et compréhensible, sa consultation nécessite peu d'efforts. D'après notre enquête, ces représentations sont largement partagées par l'ensemble des jeunes scolarisés de 11 à 25 ans et constituent des fondements de son audience.

L'acceptabilité de Wikipédia dépend en second lieu de facteurs sociaux. Sa réputation paraît être dépendante du contexte et de la nature de la tâche à accomplir. Elle semble plutôt positive pour s'informer sur des sujets en rapport avec les loisirs. Dans un cadre académique, même si les jeunes renvoient l'image d'attitudes professorales contrastées quant à sa valeur et à son intérêt, le discours dépréciatif est ressenti comme étant dominant. L'avancée dans la scolarité va ainsi de pair avec la perception accrue de risques liés à son utilisation à des fins scolaires ou universitaires. D'une part, les jeunes prennent progressivement conscience que son usage peut s'avérer pénalisant si l'enseignant a une opinion négative de l'encyclopédie. Et, d'autre part, au fil de leur cursus académique, ils deviennent davantage sensibles aux questions liées à sa fiabilité. La confiance manifestée à l'égard des supports imprimés tranche avec l'incertitude générée par le modèle éditorial collaboratif et ouvert inhérent à Wikipédia.

Ce constat invite à s'interroger sur les connaissances informationnelles que les jeunes ont sur l'encyclopédie. Quel est leur degré de connaissance de ce modèle à la fois complexe et inédit, reposant à la fois sur la possible participation de chacun, le respect de règles précises et le contrôle par d'autres contributeurs qui disposent de droits équivalents ? Ces 
connaissances évoluent-elles selon l'âge ? Ont-elles une incidence sur la confiance accordée à Wikipédia ? Autant de points de départs pour de futures investigations.

Décembre 2013

Merci aux nombreux enseignants qui nous ont aidé pour la passation du questionnaire ainsi qu'à mes collègues de l'ESPE de Toulouse, Benoît Jeunier, Josiane Mothe et André Tricot.

\section{Références bibliographiques}

[1] K. AILLERIE. Pratiques informationnelles informelles des adolescents (14-18 ans) sur le Web. Thèse en sciences de l'information et de la communication, Université Paris 13, 2011

[2] A. BÉGUIN, S. KOVACS. (dir.). Le cahier et l'écran: culture informationnelle et premiers apprentissages documentaires. Hermès science-Lavoisier, 2011

[3] J. P. BIDDIX, J.C. CHUNG, H. W. PARK. « Convenience or credibility? A study of college student online research behaviors ». The Internet and Higher Education, 2011, vol. 14, n 3, p. 175-182

[4] A. CORDIER. Imaginaires, représentations, pratiques formelles et non formelles de la recherche d'information sur Internet : le cas d'élèves de $\sigma^{e}$ et de professeurs documentalistes. Thèse en sciences de l'information et de la communication. Université Charles de Gaulle Lille III, 2011

[5] C. DIONI. Métier d'élève, métier d'enseignant à l'ère numérique. INRP, 2008

[6] A. J. FLANAGIN, M. METZGER. Kids and credibility: An empirical examination of youth, digital media use, and information credibility. MIT Press, 2010

[7] M. FOGLIA. Wikipédia : média de la connaissance démocratique ? FYP Éditions, 2008

[8] H. FRANCKE, O. SUNDIN. " Negotiating the role of sources: Educators' conceptions of credibility in participatory media ». Library \& Information Science Research, 2012, n³4, p. 169-175

[9] E. HARGITTAI, L. FULLERTON, E. MENCHEN-TREVINO, K. YATES-THOMAS. « Trust Online: Young Adults' Evaluation of Web Content ». International Journal of Communication, 2010, vol. 4, p. 468494

[10] A. J. HEAD, M. B. EISENBERG. « How today's college students use Wikipedia for course-related research ». First Monday, 2010, vol. 15, n 3

[11] B. JUANALS. La culture de l'information : du livre au numérique. Hermès science, 2003 
[12] T. JUDD, G. KENNEDY. « A five-year study of on-campus Internet use by undergraduate biomedical students ». Computers \& Education, 2010, vol. 55, n² 4, p. 1564-1571

[13] H. JULIEN, S. BARKER. « How high-school students find and evaluate scientific information: A basis for information literacy skills development ». Library \& Information Science Research, 2009, vol. $31, n^{\circ} 1$, p. $12-17$

[14] L. LE DOUARIN, H. DELAUNAY-TÉTEREL. "Le "net scolaire" à l'épreuve du "temps libre" des lycéens ». Revue Française de socio-économie, 2011, n 8, p. 103-121

[15] D. LEWANDOWSKI, U. SPREE. " Ranking of Wikipedia articles in search engines revisited: Fair ranking for reasonable quality? " Journal of the American Society for Information Science and Technology, 2011, vol. 62, n 1, p. 117-132

[16] S. LIM. « How and Why Do College Students Use Wikipedia? » Journal of the American Society for Information Science and Technology, 2009, vol. 60, n 11, p. 2189-2202

[17]B. LUYT, C. ZAINAL, O. MAYO, T. YUN. « Young people's perceptions and usage of Wikipedia ». Information Research, 2008, vol. 13, $\mathrm{n}^{\circ} 4$

[18] K. PURCELL, A. HEAPS, J. BUCHANAN, L. FRIEDRICH. How teachers are using technology at home and in their classrooms. Pew Research Center's Internet \& American Life Project, 2013

[19] X.-L. SHEN, C. M. K. CHEUNG, M. K. O. LEE. « What leads students to adopt information from Wikipedia? An empirical investigation into the role of trust and information usefulness ». British Journal of Educational Technology, 2013, vol. 44, n³, p. 502-517

[20] B. SIMONNOT. L'accès à l'information en ligne : moteurs, dispositifs et médiations. Hermès science publications-Lavoisier, 2012

[21] F. de SINGLY. Le questionnaire. $2^{\mathrm{e}}$ éd. Armand Colin, 2005

[22] O. SUNDIN, H. FRANCKE. « In search of credibility: pupils' information practices in learning environments ». Information Research, 2009, vol. 14, $\mathrm{n}^{\circ} 4$

[23] R. WALLACE, J. KUPPERMAN, J. KRAJCIK. « Science on the Web : Students online in a sixth-grade classroom ». The Journal of the learning sciences, 2000, vol. 9, n 1, p. 75-104 